\title{
Wind power application research on the fusion of the determination and ensemble prediction
}

\author{
Shi Lan, Xu Lina, and Hao Yuzhu \\ Inner Mongolia Meteorological Service Center, Hohhot 010051, China \\ Correspondence to: Shi Lan (lan_shi@sina.com)
}

Received: 14 January 2017 - Revised: 24 May 2017 - Accepted: 30 May 2017 - Published: 11 July 2017

\begin{abstract}
The fused product of wind speed for the wind farm is designed through the use of wind speed products of ensemble prediction from the European Centre for Medium-Range Weather Forecasts (ECMWF) and professional numerical model products on wind power based on Mesoscale Model5 (MM5) and Beijing Rapid Update Cycle (BJ-RUC), which are suitable for short-term wind power forecasting and electric dispatch. The single-valued forecast is formed by calculating the different ensemble statistics of the Bayesian probabilistic forecasting representing the uncertainty of ECMWF ensemble prediction. Using autoregressive integrated moving average (ARIMA) model to improve the time resolution of the single-valued forecast, and based on the Bayesian model averaging (BMA) and the deterministic numerical model prediction, the optimal wind speed forecasting curve and the confidence interval are provided. The result shows that the fusion forecast has made obvious improvement to the accuracy relative to the existing numerical forecasting products. Compared with the 0-24 $\mathrm{h}$ existing deterministic forecast in the validation period, the mean absolute error (MAE) is decreased by $24.3 \%$ and the correlation coefficient $(R)$ is increased by $12.5 \%$. In comparison with the ECMWF ensemble forecast, the MAE is reduced by $11.7 \%$, and $R$ is increased $14.5 \%$. Additionally, MAE did not increase with the prolongation of the forecast ahead.
\end{abstract}

\section{Introduction}

Because of atmospheric movements are instable, exhibiting chaotic characteristics, the deterministic prediction from a single-valued numerical weather prediction simulation contains unavoidable errors. The most important for reducing the uncertainties of numerical weather prediction simulation refers to the ensemble method, which conducts ensemble simulations by setting different initial conditions (Deppe et al., 2013; Lin et al., 2012). Ensemble forecast and multimodel consensus forecast technology is a main direction of the development of weather forecasting technology. An ensemble forecast system can reduce the prediction error and provide the spatial and temporal evolution for the probability density distribution (Liu, 2000), so it offers certain evidence to judge forecasts' credibility. The technology of multimodel consensus forecast technology is a very effective statistical post-processing method to improve the accuracy of the numerical forecast. With the development of ensemble forecast as well as its interpretation and application, more and more scholars applied them to the wind energy prediction. An improved multi-step forecasting model based on WRF ensembles and creative fuzzy systems for wind speed yields the best forecasting performance and outperforms individual ensemble members and all of the other models for comparison (Zhao et al., 2014). The Bayesian model averaging (BMA) model is used to calibrate the wind ensemble forecast, the error of wind forecast is reduced, and the coverage of the 66.7 and $90 \%$ prediction intervals is very closed with a highly concentrated forecasting probability density functions (PDFs) being produced (Baran, 2014). It is verified that a better-calibrated probabilistic forecast is provided by the BMA method compared to the raw ensemble (Sloughter et al., 2010).

A fusion forecast which can improve the forecast's accuracy and quality of time and space is developed in this paper based on ensemble forecasts of European Centre for Medium-Range Weather Forecasts (ECMWF) and the deterministic mesoscale numerical forecast named Mesoscale 


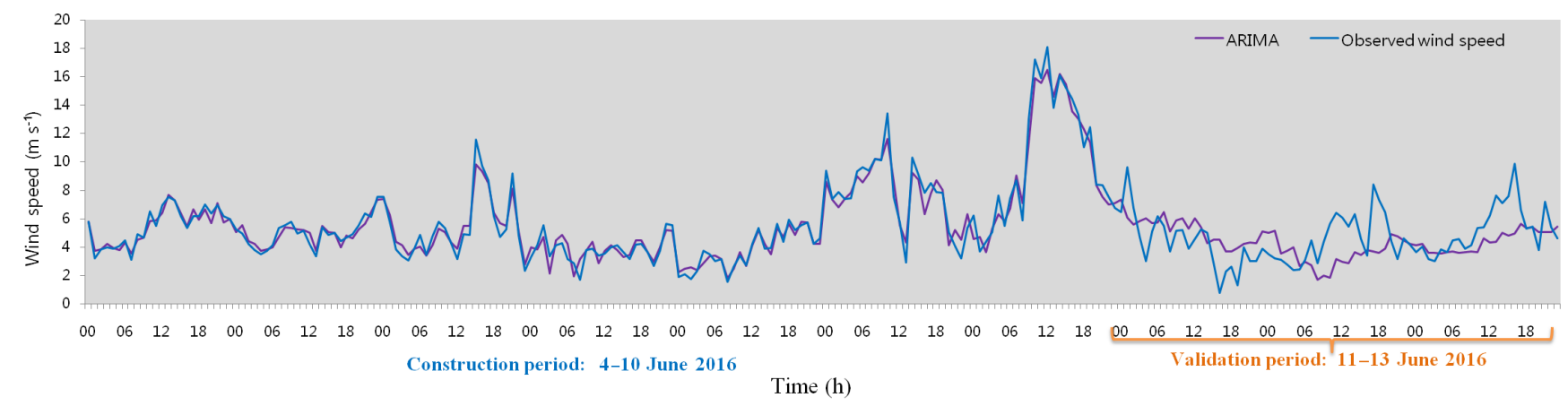

Figure 1. The contrast between the 0-72 h ARIMA downscaling forecast and the observed wind speed on 10 June 2016.

Model5 (MM5) and Beijing Rapid Update Cycle (BJ-RUC) for wind power on local. The wind speed ensemble members from ECMWF are calibrated by probability matching technique on the basis of the BMA method, and then time downscaling is done. The wind speed forecasts from MM5 and BJ-RUC are statistically corrected. Finally, the three forecasts are fused by BMA method. It fulfills the application requirements including a limited operation environment of numerical model and resource conditions, the same spatial and temporal resolution with the deterministic forecast and forecasts up to $72 \mathrm{~h}$.

\section{Data}

The studied wind farm is located in the middle of Inner Mongolia, China, and the average altitude is about $1560 \mathrm{~m}$. The terrain is higher in the north and lower in the south and the elevation difference is about $200 \mathrm{~m}$. The area of the wind farm is about $70 \mathrm{~km}^{2}$. The average annual wind speed is about $5-$ $6 \mathrm{~m} \mathrm{~s}^{-1}$ (Shi et al., 2016). The wind tower is located near the center of the wind farm and there are $5.206 \mathrm{~km}$ far away the nearest wind turbine. The wind measurement is mounted on the top of the wind tower. The model of wind turbines in the wind farm is GemesaV52-850. The hourly observed wind speed at $70 \mathrm{~m}$ from the wind tower, the wind speed forecast at $100 \mathrm{~m}$ from ECMWF, the wind speed forecast at $70 \mathrm{~m}$ and surface pressure forecast from MM5 and BJ-RUC are used. The starting forecast time is $20 \mathrm{~h}$ (China standard time) and the forecast is $72 \mathrm{~h}$ ahead. The horizontal resolution of ECMWF is $0.5^{\circ}$ and time resolution is $3 \mathrm{~h}$. The horizontal resolution of MM5 and BJ-RUC is $9 \mathrm{~km}$ and the time resolution is $1 \mathrm{~h}$. The data period is from 31 May to $31 \mathrm{Au}-$ gust 2016. Because the terrain of the wind farm is relatively flat, the influence of terrain can be ignored. The wind speed forecast at heights of 70 and $100 \mathrm{~m}$ at the location of wind tower is calculated by bilinear interpolation and the wind speed forecast at $100 \mathrm{~m}$ is fitted to the measurement data through linear relation.

\section{Method}

\subsection{Construction of BMA model and parameter calibration}

The BMA method is a statistical post-process method for inference and prediction combined with multiple statistical models. Probability forecast of a specified variable using it is the weighted average of probability prediction after deviation correction every model. The weights are the posterior probabilities of the corresponding model, representing the relative forecast skill of each model in the model training stage. The calibrated and highly concentrated forecasting PDFs can be produced and the percentage of future weather can be predicted (Zhao et al., 2014; Liu et al., 2013).

In terms of the wind speed modeling, we employ a truncated normal distribution followed by $N^{0}\left(\mu, \sigma^{2}\right)$ with a cutoff at zero in this paper and the location is assumed to be a linear function of ECMWF. The optimum training period of 18 days is tested through using the observed and forecast wind speed from 31 May to 31 August 2016. The singlevalued forecast is the sum of products of the posterior probabilities and the ensemble members. The width of the 5th and 95th percentiles was taken as the interval of wind speed distribution. The previous 18 days are taken as the training period to forecast $0-72 \mathrm{~h}$ wind speed dynamically. Additionally, the test indexes in the training period and verification period are also raised compared with ECMWF.

\subsection{Statistical correction for numerical forecast}

In order to minimize the system error of the numerical model and provide a more accurate prior probability for the BMA model, the numerical forecasts of MM5 and BJ-RUC are corrected using the method of analogue correction of model errors (ACE) (Jiang et al., 2013). The wind speed forecast at $70 \mathrm{~m}$ and surface pressure forecast from MM5 and BJ-RUC are selected as the correction factors. The model parameters are determined by conducting a sensitivity test; the time window is $2 \mathrm{~h}$, the weight of wind speed is 1 and surface pressure is 0.1 . Additionally, the sample size of the similarity 

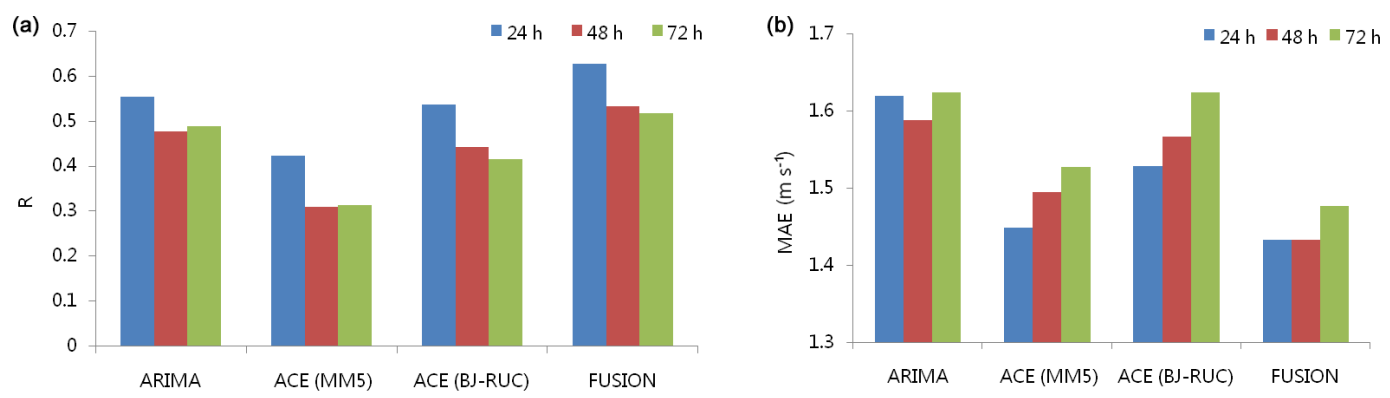

Figure 2. The contrast of the correlation coefficient (a) and mean absolute error (b) during each step.

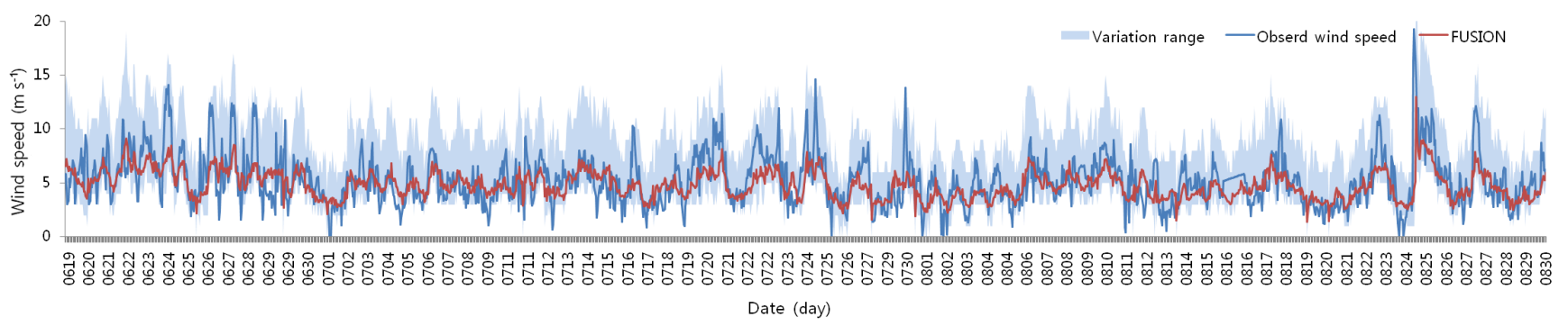

Figure 3. The contrast of $0-24 \mathrm{~h}$ single-valued forecast, variation range of wind speed and hourly observed wind speed in the verification period.

forecast is 25 . The prediction performance after correction is obviously improved compared with MM5 and BJ-RUC. The mean absolute error (MAE) of $0-24 \mathrm{~h}$ wind speed forecasts from MM5 is decreased from 1.87 to $1.53 \mathrm{~m} \mathrm{~s}^{-1}$ and that from BJ-RUC is decreased from 1.90 to $1.66 \mathrm{~m} \mathrm{~s}^{-1}$.

\subsection{Time downscaling by autoregressive integrated moving average (ARIMA)}

The time resolution of wind speed forecast from ECMWF is $3 \mathrm{~h}$, but the time resolution of BJ-RUC and MM5 is $1 \mathrm{~h}$. Therefore, the time downscaling for ECMWF from 3 to $1 \mathrm{~h}$ is required. Based on the idea of ARIMA (Wang et al., 2015; Fan, 2010), using the hourly observed wind speed from the wind tower and BMA forecast with $3 \mathrm{~h}$ time resolution, the ARIMA time downscaling model is established. The optimum simulation period of 7 days is determined by testing. The time resolution of BMA forecasting products reduces from 3 to $1 \mathrm{~h}$. After comparison, it is found that the effect of forecast after ARIMA does not reduce with the improvement of the forecast resolution and the quality of the raw BMA forecast product is ensured (Fig. 1).

\subsection{Fusion of three numerical forecasts}

We employ a truncated normal distribution with a cutoff at zero $\ldots 0(\ldots, \ldots 2)$ and the location is assumed to be a linear function of the BMA downscaling forecast and the corrected wind speed from MM5 and BJ-RUC. The fusion forecast of the wind farm is generated from the three numerical forecasts using the BMA method. The effect of the fusion forecast is higher than one of the three numerical forecasts and the mean consensus of the three models.

\section{Modeling and verification}

Using the above-mentioned methods and indicators, the model of fusion forecast is established dynamically every day. A $0-72 \mathrm{~h}$ fusion forecast and the interval of wind speed distribution are calculated. $R$ and MAE are used as indexes to test and evaluate the effect of the fusion forecasting products. The results (Fig. 2) demonstrate that $R$ rise to 0.520.63 and MAE fell to $1.43-1.48 \mathrm{~m} \mathrm{~s}^{-1}$. It is better than the BMA downscaling forecast and the corrected wind speed from MM5 and BJ-RUC. As a result, the model can effectively improve the prediction performance and the effect is obviously better than the method of statistical post processing for a single numerical forecasting model.

In Fig. 3, the contrast of 0-24h single-valued forecasts, variation range of wind speed and hourly observed wind speed in the verification period are given. It is proven that the trend of the single-valued forecast is basically the same with observed wind speed. Although the variation range of wind speed is smaller, the forecasted wind speed is located at the position of the wind tower and represents the same area as the tower's observed wind speed. The products' generation can capture the turning wave of wind speed and make up the defect of the smaller variation range of the deterministic forecast. 


\section{Summary}

The BMA method is applied to the short-term wind power prediction and the dynamic fusion forecasting model for wind farm is established, which can effectively reduce the forecasting error and improve the accuracy of the existing numerical forecasting product. Compared with the $0-24 \mathrm{~h}$ existing deterministic forecast (the average wind speed from MM5 and BJ-RUC) in the validation period, MAE is decreased by $24.3 \%$ and $R$ is increased $12.5 \%$. Compared with the ECMWF ensemble forecast (the average of 51 ensemble members), MAE is reduced by $11.7 \%$ and $R$ is increased $14.5 \%$.

The interval of wind speed distribution can capture the turning wave of wind speed to reduce the risk of short-term wind power forecasting and electric dispatch from the deterministic forecast.

Data availability. No data sets were used in this article.

Competing interests. The authors declare that they have no conflict of interest.

Special issue statement. This article is part of the special issue "16th EMS Annual Meeting \& 11th European Conference on Applied Climatology (ECAC)". It is a result of the 16th EMS Annual Meeting \& 11th European Conference on Applied Climatology (ECAC), Trieste, Italy, 12-16 September 2016.

Edited by: Sven-Erik Gryning

Reviewed by: two anonymous referees

\section{References}

Baran, S.: Probabilistic wind speed forecasting using Bayesian model averaging with truncated normal components, Comput. Stat. Data An., 75, 227-238, 2014.

Deppe, A. J., Gallus Jr., W. A., and Takle, E. S.: A WRF ensemble for improved wind speed forecasts at turbine height, Weather Forecast., 28, 212-228, 2013.

Fan, L.: Preliminary study of statistically downscaled temperature ensemble predictions in eastern China, Plateau Meteorology, 29, 392-402, 2010.

Jiang, Y., Song, L., and Cheng, X.: An integrated and revised method of forecasting wind speed for wind farms, Resources Science, 35, 673-680, 2013.

Lin, W., Wang, J., Zhang, W., Guo, Z., Chi, D., and Zhang, Y.: Program of wind speed prediction based on numerical simulation with intelligent optimization algorithm, Climatic Environ. Res., 17, 646-658, 2012.

Liu, J.: Ensemble prediction ushered in new era in numerical weather prediction, Meteorological Monthly, 26, 21-25, 2000.

Liu, J., Xie, Z., Zhao, L., and Jia, B.: BMA probabilistic forecasting for the 24-h TIGGE multi-model ensemble forecasts of surface air temperature, Chinese Journal of Atmospheric Sciences, 37 , 43-53, 2013 (in Chinese).

Shi, L., Xu, L., and Hao, Y.: The correction of wind speed forecast in a wind farm based on wind turbine groups of the high correlation of wind speed, Quarterly Journal of Applied Meteorology, 27, 506-512, 2016.

Sloughter, M. L., Gneiting, T., and Raftery, A. E.: Probabilistic Wind Speed Forecasting Using Ensembles and Bayesian Model Averaging, J. Am. Stat. Assoc., 105, 25-35, 2010.

Wang, L., Zhao, Y., Yang, X., Ma, J., Huang, T., and Gao, H.: Prediction of Air Quality in Lanzhou Using Time Series Model and Residual Control Chart, Plateau Meteorology, 34, 230-236, 2015.

Zhao, L., Liu, Y., Dang, H., Jiang, D., Duan, Q., Wang, B., Bai, X., and Liang, L.: The progress on application of ensemble prediction to flood forecasting, Journal of Applied Meteorological Science, 6, 641-653, 2014. 